\title{
Severe reduction of free-form ADAMTS13, unbound to von Willebrand factor, in plasma of patients with HELLP syndrome
}

\author{
Yoko Yoshida, ${ }^{1, \star}$ Masanori Matsumoto, ${ }^{1}$ Hideo Yagi, ${ }^{1, \star}$ Ayami Isonishi, ${ }^{1}$ Kazuya Sakai, ${ }^{1}$ Masaki Hayakawa, ${ }^{1}$ Yuji Hori, ${ }^{2}$ Toshiyuki Sado, ${ }^{3}$ \\ Hiroshi Kobayashi, ${ }^{3}$ and Yoshihiro Fujimura ${ }^{2, \star}$ \\ ${ }^{1}$ Department of Blood Transfusion Medicine, Nara Medical University, Kashihara, Japan; ${ }^{2}$ Japanese Red Cross Kinki Block Blood Center, Ibaraki, Japan; and ${ }^{3}$ Department of \\ Obstetrics and Gynecology, Nara Medical University, Kashihara, Japan
}

\section{Key Points}

- Severely decreased ADAMTS13 unbound to VWF may play a key role in the pathogenesis of HELLP syndrome.

- A qualitative ADAMTS13 assay may be important for diagnosing HELLP syndrome.

\section{Introduction}

\begin{abstract}
Hemolysis, elevated liver enzymes, and low platelet count (HELLP) syndrome, a life-threatening complication during pregnancy, is a type of thrombotic microangiopathy generally associated with preeclampsia. Because of its clinical similarity to thrombotic thrombocytopenic purpura, ${ }^{1}$ studies of HELLP syndrome have focused on von Willebrand factor (VWF) and its cleaving protease a disintegrinlike and metalloproteinase with thrombospondin type 1 motifs 13 (ADAMTS13). ${ }^{2-4}$ Several groups reported that patients with HELLP syndrome showed a marked increase of plasma VWF antigen (VWF:Ag) (200\%-500\%) with a full set of VWF multimers (VWFMs) as compared with normal pregnant women $(\sim 180 \%),{ }^{2,3,5}$ Inversely, patients with HELLP syndrome had low or normal ADAMTS13 activity (ADAMTS13:AC) $(20 \%-70 \%))^{2-4}$ These subnormal ADAMTS13:AC levels are thought to be able to eliminate preexisting unusually large VWFMs, which are responsible for platelet hyperaggregation under high shear stress. ${ }^{6}$ However, the mechanism underlying increased VWF:Ag levels in HELLP syndrome is still not elucidated. Previously, we conducted a qualitative analysis of ADAMTS13 antigen (ADAMTS13:AG) in normal plasma, using isoelectrofocusing (IEF) with a large-pore agarose-acrylamide composite gel, and visualized at least 2 forms of plasma ADAMTS13, bound ( $\sim 5 \%$ of total) and unbound to VWF. ${ }^{7}$ Subsequently, we demonstrated that these 2 forms of ADAMTS13 differentially modulated VWF-dependent platelet aggregation under high shear stress in a dose-dependent manner. ${ }^{7}$ Here, we performed a comparative study involving qualitative IEF analysis of ADAMTS13:AG in plasma serially obtained from 8 patients with HELLP syndrome and plasma from normal pregnant women or those with preeclampsia.
\end{abstract}

\section{Methods}

This study analyzed 128 normal pregnant women, 5 patients with preeclampsia, and 8 patients with HELLP syndrome, under approval of the ethics committee of Nara Medical University. All participants gave written informed consent. Plasma levels of ADAMTS13:AC and VWF:Ag were determined by chromogenic ADAMTS13-act-enzyme-linked immunosorbent assay (ELISA) (Kainos, Tokyo, Japan) ${ }^{8}$ and a sandwich ELISA using a rabbit polyclonal anti-human VWF antibody (Dako, Glostrup, Denmark), ${ }^{9}$ respectively. IEF using a large-pore agarose-acrylamide composite gel was performed as previously reported ${ }^{7,10}$; details are presented in supplemental Methods. In normal citrated plasma, 3 ADAMTS13:AG bands were detected ${ }^{7,10}$ : band I (isoelectric point [pl], 4.9-5.6) was free (unbound) ADAMTS13:AG, band III (pl, 7.0-7.5) was ADAMTS13:AG complexed with high-molecular-weight VWFMs, but band II (pl, 5.8-6.7) was undetermined.

All data are expressed as medians unless otherwise noted. Differences in VWF:Ag, ADAMTS13:AC, platelet counts, and amounts of ADAMTS13:AG bands between all groups were evaluated using the Kruskal-Wallis test, followed by the Steel-Dwass test on each pair. Statistical analysis was performed using JMP 11.0 statistical software (SAS Institute, Cary, NC). A $P$ value below .05 was considered significant.

\section{Results and discussion}

The clinical characteristics and laboratory findings of 8 patients with HELLP syndrome are summarized in supplemental Table 1. In normal pregnancy, plasma VWF:Ag levels began increasing in the second
Submitted 14 March 2017; accepted 13 July 2017. DOI 10.1182/ bloodadvances. 2017006767.

*Y.Y., H.Y., and Y.F. contributed equally to this work.
The full-text version of this article contains a data supplement. (C) 2017 by The American Society of Hematology 
Figure 1. Plasma levels of VWF:Ag and ADAMTS13:AC, the platelet counts during normal pregnancy, and changes of plasma levels of VWF:Ag and ADAMTS13:AC in patients with preeclampsia and patients with HELLP syndrome. (A) Plasma levels of VWF:Ag and ADAMTS13:AC, and platelet counts, in normal pregnant women. Plasma VWF:Ag levels increased during pregnancy and peak levels were found in the third trimester of normal pregnancy, but these decreased rapidly in the postpartum period (top panel). Plasma ADAMTS13:AC levels decreased progressively, even after delivery (middle panel). Platelet counts slightly decreased during pregnancy but were within the normal range (bottom panel). (B) Comparison of plasma VWF:Ag and ADAMTS13:AC between normal pregnant women, pregnant women with preeclampsia, and women with HELLP syndrome in the postpartum period. Plasma levels of VWF:Ag in women with HELLP syndrome were significantly higher than those in normal pregnant women in the postpartum period (top panel, $352.0 \%$ vs $179.5 \%, P<.001)$. There were no statistical differences in ADAMTS13:AC between the 3 groups (bottom panel: normal pregnancy, 52.1\%; preeclampsia, 50.9\%; and HELLP syndrome, 50.5\%). N.S., not significant.
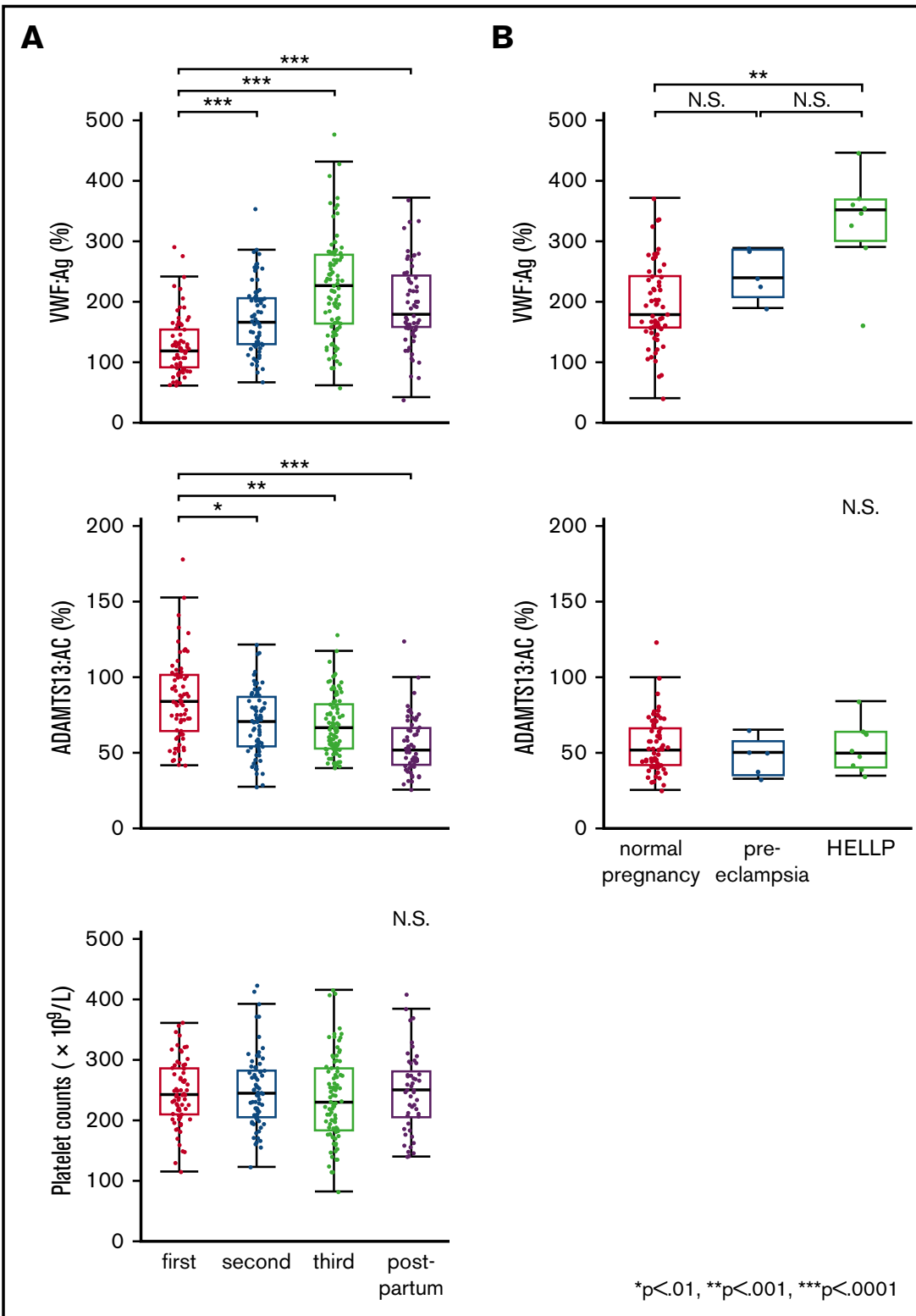

${ }^{*} p<.01,{ }^{\star *} p<.001,{ }^{* \star *} p<.0001$ trimester (166.0\%). In the third trimester, levels (227.0\%) were almost twice as high as in the first (119.5\%). One week after delivery, levels dropped rapidly (179.5\%) (Figure 1A top panel). Plasma ADAMTS13:AC levels decreased progressively during pregnancy (first trimester, 84.3\%; second, 70.8\%; third, 66.8\%), and their reduction continued after delivery (52.1\%) (Figure $1 \mathrm{~A}$ middle panel). Platelet counts were almost unchanged and stayed within normal ranges during pregnancy and postpartum (Figure 1A bottom panel). We then compared postpartum plasma levels of VWF:Ag and ADAMTS13:AC between normal pregnant women, patients with preeclampsia, and patients with HELLP syndrome. As shown in Figure 1B (top panel), preeclampsia and HELLP syndrome were associated with increased plasma VWF:Ag levels compared with normal pregnancy. Differences in VWF:Ag levels reached statistical significance between normal pregnancy and HELLP syndrome $(179.5 \%$ vs $352.0 \% ; P<.001)$. Nevertheless, no significant changes in ADAMTS13:AC were found between the
3 groups (Figure 1B bottom panel). The IEF assay of ADAMTS13: AG showed that the median amounts of bands I, II, and III measured by densitometric analysis in normal plasma were 53.5\%, 38.0\%, and $8.5 \%$, respectively. Figure $2 \mathrm{~A}$ shows the IEF patterns of 5 normal pregnant women (NP1-5) whose plasma was serially obtained in each of the 3 trimesters, and soon after delivery. No marked changes were immediately apparent, but densitometric analysis indicated that the amounts of band I gradually decreased during pregnancy (first trimester, 44.5\%; second, 40.5\%; third, $36.6 \%$; postpartum, $38.9 \%$ ). IEF patterns of preeclampsia patients (Figure 2B PE1-5) were almost the same as those of normal pregnant women, with the exception of postpartum day 3 in a PE3 patient. Interestingly, in HELLP syndrome patients (Figure $2 \mathrm{C} \mathrm{HS1-8),}$ band I was markedly reduced without exception, especially from day 1 to day 5 postpartum (Figure 2C) when the median value was significantly lower than that in normal pregnancy $(10.4 \%$ vs $38.9 \%$; $P<$.05). However, there were no statistical differences in amounts 


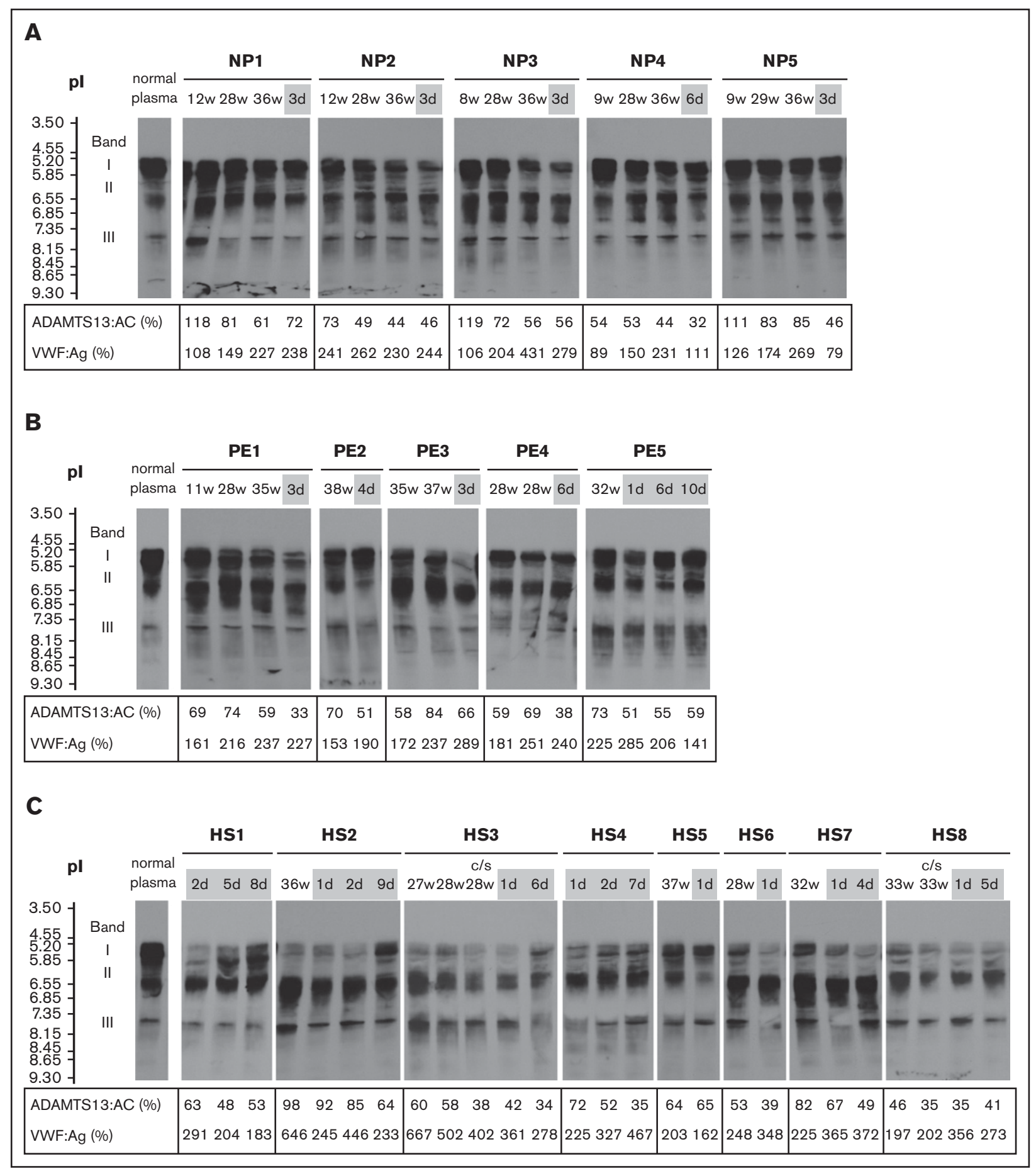

Figure 2. Separation of plasma ADAMTS13:AG by IEF. The week of gestation or the day after delivery is shown at the top of the figure, and plasma levels of ADAMTS13:AC and VWF:Ag are shown at the bottom. The IEF pattern of ADAMTS13:AG in normal plasma is indicated on the left. The number of days postpartum is indicated with a gray background. IEF analysis of each sample was performed in a different gel. (A) In all 5 normal pregnant women (NP), band I of ADAMTS13 (ie, the unbound form of ADAMTS13) slightly decreased during pregnancy. (B) Five patients with preeclampsia (PE) showed no marked changes of IEF patterns, and only patient 3 (PE3) showed a reduction of band I in the postpartum period. (C) All 8 patients with HELLP syndrome (HS) showed a severe reduction of band I, especially in the postpartum period. c/s, cesarean section.

of band I between preeclampsia and normal pregnancy $(29.2 \%$ vs $38.9 \%$ ). Band I also showed a severe decrease in the third trimester in 5 patients (HS2, HS3, HS6, HS7, and HS8), but recovered at 7 to 9 days postpartum in 2 patients (HS2 and HS4). Amounts of band III in HELLP syndrome were significantly higher than normal pregnancy $(19.5 \%$ vs $7.7 \% ; P<.05)$, and those of band II were also higher but not significantly. We previously showed that band I was the unbound form of ADAMTS13:AG, band III was 
ADAMTS13:AG complexed with high-molecular-weight VWFMs, and band II remained undetermined. ${ }^{7}$ Furthermore, we demonstrated that bands I (and II) and band III were separated by cryoprecipitation, and inhibited VWF-dependent platelet aggregation under high shear stress via different mechanisms by cryosupernatant including bands I and II in the initial phase, and by cryoprecipitation including band III in the later phase, both in a dose-dependent manner. ${ }^{7}$ Interestingly, the degree of inhibition of platelet aggregation at each end point was almost the same, suggesting that the impact on VWFMs cleavage was fast-acting by bands I (and II), and slow-acting by band III. Therefore, we speculate that a severe reduction of band $I$, resulting in a lack of fast-acting ADAMTS13, may play a key role in the pathogenesis of HELLP syndrome. A limitation of this study was that the patient placentas were not examined pathologically. A recent study showed that syncytiotrophoblast cells in the placenta adopt vascular characteristics such as the presence of VWF, CD31 markers, adhesion molecules, and coagulation components. ${ }^{11,12}$ Although further studies are needed, several lines of evidence suggest that syncytiotrophoblasts constitutively or upon stimulation secrete VWF into the intervillous space, which can supply the maternal circulation with additional VWF. In patients with HELLP syndrome, severe reduction of band I might reflect the consumption of the unbound form of ADAMTS13 due to unusually large VWFMs, which are excessively generated during pregnancy. Therefore, replenishment of the unbound form of ADAMTS13 might be a promising option for the treatment of HELLP syndrome.

Although the number of patients was limited, this study clearly showed a severe reduction of band I of ADAMTS13:AG in HELLP syndrome, but not in preeclampsia. However, whether a severe reduction of band $\mathrm{I}$ is found in larger patient groups with markedly increased plasma VWF:Ag levels remains unaddressed. Furthermore, we previously showed that ADAMTS13 forms a noncovalent complex with VWF in plasma that may become dissociated by rigorous maneuvers. ${ }^{7}$ The IEF method minimizes this risk, and might therefore be used as a high-throughput assay.

As a consequence, our findings emphasize the importance of both qualitative and quantitative assessment of plasma ADAMTS13:AG in HELLP syndrome.

\section{Acknowledgments}

This work was supported by research grants from the Ministry of Health, Labour and Welfare of Japan; the Takeda Medical Foundation; and the Japan Society for the Promotion of Science.

\section{Authorship}

Contribution: Y.Y. performed experiments, collected and analyzed the data, and wrote the manuscript; M.M and H.Y. provided advice, interpreted the data, and wrote the manuscript; A.I. and K.S. performed experiments; M.H. and Y.H. provided technical assistance; T.S. and H.K. provided advice and assisted with sample collection; and Y.F. conceived of and designed the study, interpreted the data, and wrote the manuscript.

Conflict-of-interest disclosure: M.M. is on clinical advisory boards for Baxalta, Alexion, and Ablynx. M.M. and Y.F. have shared patent rights of ADAMTS13-act-ELISA with Alfresa Pharma. The remaining authors declare no competing financial interests.

ORCID profiles: M.M., 0000-0002-7243-3126.

Correspondence: Masanori Matsumoto, Department of Blood Transfusion Medicine, Nara Medical University, Shijyo-cho 840, Kashihara, Nara 634-8522, Japan; e-mail: mmatsumo@naramed-u. ac.jp.

\section{References}

1. Young BC, Levine RJ, Karumanchi SA. Pathogenesis of preeclampsia. Annu Rev Pathol. 2010;5:173-192.

2. Lattuada A, Rossi E, Calzarossa C, Candolfi R, Mannucci PM. Mild to moderate reduction of a von Willebrand factor cleaving protease (ADAMTS-13) in pregnant women with HELLP microangiopathic syndrome. Haematologica. 2003;88(9):1029-1034.

3. Hulstein JJ, van Runnard Heimel PJ, Franx A, et al. Acute activation of the endothelium results in increased levels of active von Willebrand factor in hemolysis, elevated liver enzymes and low platelets (HELLP) syndrome. J Thromb Haemost. 2006;4(12):2569-2575.

4. Pourrat O, Coudroy R, Pierre F. ADAMTS13 deficiency in severe postpartum HELLP syndrome. Br J Haematol. 2013;163(3):409-410.

5. Sánchez-Luceros A, Meschengieser SS, Marchese C, et al. Factor VIII and von Willebrand factor changes during normal pregnancy and puerperium. Blood Coagul Fibrinolysis. 2003;14(7):647-651.

6. Barbot J, Costa E, Guerra M, et al. Ten years of prophylactic treatment with fresh-frozen plasma in a child with chronic relapsing thrombotic thrombocytopenic purpura as a result of a congenital deficiency of von Willebrand factor-cleaving protease. Br J Haematol. 2001;113(3):649-651.

7. Hori Y, Hayakawa M, Isonishi A, Soejima K, Matsumoto M, Fujimura Y. ADAMTS13 unbound to larger von Willebrand factor multimers in cryosupernatant: implications for selection of plasma preparations for thrombotic thrombocytopenic purpura treatment. Transfusion. 2013;53(12):3192-3202.

8. Kato S, Matsumoto M, Matsuyama T, Isonishi A, Hiura H, Fujimura Y. Novel monoclonal antibody-based enzyme immunoassay for determining plasma levels of ADAMTS13 activity. Transfusion. 2006;46(8):1444-1452.

9. Bartlett A, Dormandy KM, Hawkey CM, Stableforth P, Voller A. Factor-VIII-related antigen: measurement by enzyme immunoassay. BMJ. 1976;1 (6016):994-996.

10. Isonishi A, Bennett CL, Plaimauer B, Scheiflinger F, Matsumoto M, Fujimura Y. Poor responder to plasma exchange therapy in acquired thrombotic thrombocytopenic purpura is associated with ADAMTS13 inhibitor boosting: visualization of an ADAMTS13 inhibitor complex and its proteolytic clearance from plasma. Transfusion. 2015;55(10):2321-2330.

11. Lanir N, Aharon A, Brenner B. Procoagulant and anticoagulant mechanisms in human placenta. Semin Thromb Hemost. 2003;29(2):175-184.

12. Zhou Y, Damsky CH, Fisher SJ. Preeclampsia is associated with failure of human cytotrophoblasts to mimic a vascular adhesion phenotype. One cause of defective endovascular invasion in this syndrome? J Clin Invest. 1997;99(9):2152-2164. 